\title{
An Experimental Test of the
} Impact of Overconfidence and Gender on Trading Activity

Richard Deaves (McMaster)

Erik Lüders (Pinehurst Capital)

Guo Ying Luo (McMaster)

Presented at the Federal Reserve Bank of Atlanta, September 2005 


\section{Are people wise?}

$\square$ Socrates: "I know nothing except the fact of my ignorance."

$\square$ Abundant psychometric evidence indicates that few of us are wise in the sense of Socrates.

$\square$ We think we are a lot smarter than we really are: we are overconfident in our knowledge. 


\section{Miscalibration}

$\square \quad$ Calibration tests: Individuals are asked to construct $90 \%$ confidence intervals for currently (or soon) knowable magnitudes (such as the height of Mount Everest, or the level of the Dow in a month).

$\square$ Percentage of individuals usually markedly below $90 \%$ produce intervals that bracket the true answer.

$\square$ Or if a single individual is asked a series of such questions, once again it is commonplace for only $40-50 \%$ of her intervals to be "right."

$\square$ References: see Lichtenstein et al (1982), Yates (1990), Keren (1991) and McClelland and Bolger (1994)). 


\section{Other strains of \\ overconfidence}

$\square$ Better-than-average effect: Most of us say that we are better than average drivers.

$\square$ Illusion of control: Most of us think we will know when the bubble will be about to burst implying we should leave the market.

$\checkmark$ In our paper we concentrate on miscalibration.

$\square$ Reason: theoretical models are closest to this approach. 


\section{Theoretical models of overconfidence}

Odean (1998), Benos (1998), Caballé and Sákovics (1998), Kyle and Wang (1997), Wang (1998, 2001), Daniel, Hirshleifer and Subrahmanyam (1998, 2001) and Gervais and Odean (2001)).

$\square$ Unanimous in predicting that the greater is the level of (individual or market-wide) overconfidence, the greater is the level of trading activity.

$\square$ Intuition: the more certain you are of your view the less credence you will accord those of others and the more likely you will be to transact at a price perceived favorable to your view. 


\section{Odean (1998)}

$\square$ Traders differ in self-perception of variance of signals of terminal dividend.

$\square$ Several periods to trade and observe signals (of true dividend) and prices.

$\checkmark$ Terminal dividend distribution:

$$
\tilde{v} \sim N\left(\bar{v}, h_{v}^{-1}\right)
$$

$\square$ Noisy signals:

$y_{t i}=\tilde{v}+\widetilde{\varepsilon}_{t m}$ where $\widetilde{\varepsilon}_{t m} \sim N\left(0, h_{\varepsilon}^{-1}\right)$ 


\section{Odean (1998) cont.}

$\square$ But traders perceive their signals to be more precise than they really are.

$\square$ Resulting proposition: When traders are price takers, expected volume increases as overconfidence increases. 


\section{Barber and Odean (2000)}

$\square$ In a study of over 60,000 households during 1991-96, they find that those trading the most frequently earned an average annual return of $11.4 \%$ vs. the market's $17.9 \%$.

$\square$ Only conjecture to posit that it is the more overconfident traders who are damaging their portfolios through excessive trading.

$\square$ In naturally-occurring markets we do not observe overconfidence!

$\square$ This is why an experimental setting is appropriate. 


\section{Survey-based or}

\section{experimental antecedents}

$\square$ Glaser and Weber (2003) combine naturally-occurring market data with information elicited from a survey.

- Using trading data from online brokerage accounts and psychometric data obtained from the same group of investors who responded to an online questionnaire, they correlate various measures of trading activity with a number of metrics of overconfidence.

- They find little correlation between these OC metrics.

- Those who are most subject to the better-thanaverage effect trade more.

- Little corresponding evidence for those who are most overconfident based on calibration tests. 


\section{Survey-based or experimental antecedents ii.}

$\square$ Biais et al (2002):

- They consider impact of two psychological traits, overconfidence based on calibration tests, and selfmonitoring, namely the disposition to attend to social cues and appropriately adjust behavior.

- Both of these are measured prior to the participation of students in a series of trading sessions.

- They find that while overconfidence does not lead to an increase in trading intensity, it does serve to significantly reduce profits. 


\section{Survey-based or experimental antecedents iii.}

$\square$ Kirchler and Maciejovsky (2002):

- They investigate overconfidence in a market setting by focusing on two strains of overconfidence, miscalibration and the difference between objective accuracy and subjective certainty, and conclude that individuals are not consistently overconfident.

- They also find that sometimes the proxies designed to measure overconfidence generate inconsistent conclusions, with individuals sometimes exhibiting overconfidence and at other times underconfidence. 


\section{How we extend and improve on previous work}

1. Miscalibration has previously been measured inadequately.

- Biais et al (2002):

ㅁ 10 questions -- leading to a perhaps too low signal to noise ratio.

Trading sessions are incentivized with grades rather than cash.

- Kirchler and Maciejovsky (2002):

Participants must understand market psychology.

- Glaser and Weber (2003):

10 questions.

$\square \quad$ Internet-based survey - answers could have been looked up.

- Deaves, Lüders and Luo (2003):

20 questions.

$\square \quad$ Calibration test is administered.

$\square \quad$ Participants incentivized with cash.

$\square \quad$ No need to understand investment psychology. 


\section{How we extend and improve on previous work ii.}

$\square \quad$ 2. We examine impact of gender on trading behavior in a more systematic fashion than before.

- Some evidence that men are apparently more predisposed to overconfidence than are women (Lundeberg, Fox and Punccohar (1994)).

- May be partly due to the fact that self-attribution bias is greater for men than for women (Beyer (1990)).

- Barber and Odean (2001) supply corroboration in the context of investment behavior.

They find that men trade $45 \%$ more than women, and this results in their portfolios earning lower riskadjusted returns.

- On the other hand, Atkinson, Baird and Frye (2001) produce contrary evidence, finding that female fixed-income portfolio managers do no better (nor any worse) than their male counterparts. 


\section{How we extend and improve on previous work iii.}

$\square \quad 3$. We design an approach that provides a reason for overconfident traders to think that their signals are more accurate.

- Previously where private information is provided, there are either no differences in signal quality (Biais et al (2002)) or signals are randomly assigned (Kirchler and Maciejovsky (2002)).

- But why should an overconfident trader think that her private information is more accurate than the next trader's?

- Here subjects are told on arriving for the experimental sessions that the more accurate were their responses at the pre-experimental questionnaire session the more accurate will be their information signals.

- Even if there is no difference between individuals in terms of knowledge, overconfident people will tend to think that their answers are more accurate. 


\section{Hypotheses}

$\square$ Hypothesis 1: Overconfidence leads to increased trading activity.

$\square$ Hypothesis 2: Controlling for overconfidence, women trade less than men. 


\section{Experimental design}

8 sessions: 2 tranches of 4 sessions in 2 locations

Why 2 locations?

- Subjects first fill out an administered preexperimental questionnaire -- measures calculated that are used to assign individuals to sessions and to ascertain how informative their information signals will be.

- This however only becomes apparent to students when they show up for the experimental sessions.

- Once we have conducted such a preexperimental questionnaire session followed by a "tranche" of experimental sessions, to repeat the same procedure in the same location would likely lead to contamination because of information leakage. 


\section{Experimental design ii.}

$\square 108$ students attended preexperimental questionnaire sessions:

- 47 in Canada (23 females) and 61 in Germany (18 females).

- Canadian sessions were stratified by gender holding OC constant.

- German sessions were stratified by OC holding gender mix constant. 
Table 1: Taxonomy of sessions

\begin{tabular}{|c|c|c|c|}
\hline $\begin{array}{c}\text { Session } \\
\text { number }\end{array}$ & Location & Gender & OC level \\
\hline $\mathbf{1}$ & Canada & Female & Mixed \\
\hline 2 & Canada & Male & Mixed \\
\hline 3 & Canada & Female & Mixed \\
\hline 4 & Canada & Male & Mixed \\
\hline 5 & Germany & Mixed & Low Overconfidence \\
\hline 6 & Germany & Mixed & High Overconfidence \\
\hline 7 & Germany & Mixed & High Overconfidence \\
\hline 8 & Germany & Mixed & Low Overconfidence \\
\hline
\end{tabular}




\section{Experimental sessions}

$\square \quad$ Instructional phase followed by trading simulation.

$\square \quad 12$ single-period markets:

- 5 minutes each

- Traders endowed with 4 shares and cash

- Dividend paid at end of period (no dividends revealed till end)

- Noisy private signals provided

- Average signal was true dividend

- Traders told that their signals would be more informative the more accurate were their CI responses during pre-experimental questionnaire session

- Before trading began dividend predictions were made and recorded

$\square$ One market randomly chosen at end as payout market. 


\section{FTS trading platform}

$\square \quad$ Markets conducted in computerized environments using Financial Trading System (FTS) platform.

$\square$ Computerized double auction market program allows subjects to transact in real time over a number of market periods.

$\square \quad$ Participants can post bids and asks, or act as price-takers in accepting the best bids or asks posted by others.

$\square$ Traders can begin with endowments made up of cash and securities, and the program automatically updates portfolios after transactions and dividend payments.

$\square$ Order book was set at a depth of one which means that posted orders were erased by better bids and asks.

$\square$ Traders were permitted to transact only one share at a time. 


\section{Table 2: Mean calibration-based overconfidence for various}

subgroups - Calibration session participants

This table displays the mean level of calibration-based overconfidence (as well as the minimum, maximum and the standard deviation) for various subsamples. A well-calibrated individual would have $10 \%$ of the correct answers of the questionnaire not contained inside her intervals, implying $\mathrm{OC}=0.1$. Very overconfident people can reach $\mathrm{OC}=1$ while the minimum value by definition is $\mathrm{OC}=0$. The number of subjects in each subsample is also given. The $\mathrm{p}$-values are shown in parentheses. P-values are calculated vs. the maintained hypothesis of 0.10 (neither overconfidence nor underconfidence), except in the case of differences where the maintained hypothesis is a zero difference.

\begin{tabular}{|c|c|c|c|c|c|}
\hline OBS & Group & Meam & Min. & Max. & SD \\
\hline 108 & All & $\begin{array}{r}0.775 \\
(0.00) \\
\end{array}$ & 0.45 & 1.00 & 0.1237 \\
\hline 67 & Male & $\begin{array}{l}0.760 \\
(0.00)\end{array}$ & 0.45 & 0.95 & 0.1306 \\
\hline 41 & Female & $\begin{array}{r}0.800 \\
(0.00) \\
\end{array}$ & 0.50 & 1.00 & 0.1084 \\
\hline 47 & Canadian & $\begin{array}{r}0.845 \\
(0.00)\end{array}$ & 0.70 & 0.95 & 0.0534 \\
\hline 61 & German & $\begin{array}{r}0.721 \\
(0.00) \\
\end{array}$ & 0.45 & 1.00 & 0.1355 \\
\hline 24 & Canadian male & $\begin{array}{c}0.854 \\
(0.00)\end{array}$ & 0.75 & 0.95 & 0.0046 \\
\hline 23 & Canadian female & $\begin{array}{l}0.835 \\
(0.00) \\
\end{array}$ & 0.70 & 0.95 & 0.0592 \\
\hline 43 & German male & $\begin{array}{r}0.707 \\
(0.00)\end{array}$ & 0.45 & 0.95 & 0.1330 \\
\hline 18 & German female & $\frac{0.756}{(0.00)}$ & 0.50 & 1.00 & 0.1392 \\
\hline - & Male vs. female diff & $\begin{array}{l}-0.040 \\
(0.101)\end{array}$ & - & - & - \\
\hline - & $\begin{array}{l}\text { Canada vs. Germany } \\
\text { diff. }\end{array}$ & $\begin{array}{c}0.123 \\
(0.000)\end{array}$ & - & - & - \\
\hline - & $\begin{array}{l}\text { Male vs. female diff. - } \\
\text { Canada }\end{array}$ & $\begin{array}{r}0.019 \\
(0.217) \\
\end{array}$ & - & - & - \\
\hline - & $\begin{array}{l}\text { Male vs. female diff. - } \\
\text { Germany }\end{array}$ & $\begin{array}{l}-0.049 \\
(0.204) \\
\end{array}$ & - & - & - \\
\hline- & $\begin{array}{c}\text { Canada vs. Germany -- } \\
\text { males }\end{array}$ & $\begin{array}{l}0.147 \\
(0.000)\end{array}$ & - & - & - \\
\hline - & $\begin{array}{c}\text { Canada vs. Germany - } \\
\text { females }\end{array}$ & $\begin{array}{l}0.079 \\
(0.018)\end{array}$ & - & - & - \\
\hline
\end{tabular}


Table 3: Mean calibration-based overconfidence for various subgroups - Trading session participants

This table displays the mean level of calibration-based overconfidence (as well as the minimum, maximum and the standard deviation) for various subsamples. A well-calibrated individual would have $10 \%$ of the correct answers of the questionnaire not contained inside her intervals, implying $\mathrm{OC}=0.1$. Very overconfident people can reach $\mathrm{OC}=1$ while the minimum value by definition is $\mathrm{OC}=0$. The number of subjects in each subsample is also given. The $\mathrm{p}$-values are shown in parentheses. P-values are calculated vs. the maintained hypothesis of 0.10 (neither overconfidence nor underconfidence), except in the case of differences where the maintained hypothesis is a zero difference.

\begin{tabular}{|c|c|l|l|l|l|}
\hline OBS & Group & Mean & Min. & Max. & SD \\
\hline 64 & All & $\begin{array}{c}0.781 \\
(0.000)\end{array}$ & 0.45 & 1.00 & 0.1402 \\
\hline 34 & Male & $\begin{array}{c}0.766 \\
(0.000)\end{array}$ & 0.45 & 0.95 & 0.1570 \\
\hline 30 & Female & $\begin{array}{c}0.798 \\
(0.000)\end{array}$ & 0.50 & 1.00 & 0.1185 \\
\hline 32 & Canadian & $\begin{array}{c}0.842 \\
(0.000)\end{array}$ & 0.70 & 0.95 & 0.0556 \\
\hline 32 & German & $\begin{array}{c}0.720 \\
(0.000)\end{array}$ & 0.45 & 1.00 & 0.1708 \\
\hline 16 & $\begin{array}{c}\text { Canadian male } \\
0.847 \\
(0.000)\end{array}$ & 0.75 & 0.95 & 0.0531 \\
\hline 16 & $\begin{array}{c}\text { Canadian female } \\
0.837 \\
(0.000)\end{array}$ & $\begin{array}{c}0.694 \\
(0.000)\end{array}$ & 0.70 & 0.95 & 0.0592 \\
\hline 14 & $\begin{array}{c}\text { German male female } \\
\mathbf{0 . 7 5 4} \\
(0.000)\end{array}$ & 0.50 & 1.00 & 0.1525 \\
\hline- & $\begin{array}{c}\text { Male vs. female } \\
\text { diff. }\end{array}$ & $\begin{array}{c}-0.032 \\
(0.364)\end{array}$ & - & - & - \\
\hline- & $\begin{array}{c}\text { Canada vs. Germany } \\
\text { diff. }\end{array}$ & $\begin{array}{c}0.122 \\
(0.000)\end{array}$ & - & - & - \\
\hline- & $\begin{array}{c}\text { Male vs. female diff. } \\
- \text { Canada }\end{array}$ & $\begin{array}{c}0.009 \\
(0.641)\end{array}$ & - & - & - \\
\hline- & $\begin{array}{c}\text { Male vs. female diff. } \\
- \text { Germany }\end{array}$ & $\begin{array}{l}-0.059 \\
(0.340)\end{array}$ & - & - & - \\
\hline- & $\begin{array}{c}\text { Canada vs. Germany } \\
- \text { males }\end{array}$ & $\begin{array}{l}0.153 \\
(0.003)\end{array}$ & - & - & - \\
\hline- & $\begin{array}{c}\text { Canada vs. Germany } \\
- \text { females }\end{array}$ & $\begin{array}{l}0.083 \\
(0.051)\end{array}$ & - & - & - \\
\hline
\end{tabular}


Table 4: Mean trading activity for various subgroups

This table displays the mean trading volume (total number of trades during the 12 single-period markets) as well as the minimum, maximum and standard deviation for various subsamples. The number of subjects in each subsample is also given. We also give differences in trading volume between various subgroups, with p-values shown in parentheses where the maintained hypothesis is a zero difference.

\begin{tabular}{|c|l|l|l|l|l|}
\hline Trader & OBS & MEAN & MIN & MAX & SD \\
\hline All & 64 & 44.594 & 7 & 130 & 26.089 \\
\hline Male & 34 & 45.088 & 14 & 130 & 24.009 \\
\hline Female & 30 & 44.033 & 7 & 112 & 28.671 \\
\hline Canadian & 32 & 55.438 & 18 & 130 & 30.783 \\
\hline German & 32 & 33.750 & 7 & 63 & 13.887 \\
\hline Canadian male & 16 & 51.875 & 18 & 130 & 30.869 \\
\hline Canadian female & 16 & 59.000 & 19 & 112 & 31.279 \\
\hline German male & 18 & 39.056 & 14 & 63 & 14.010 \\
\hline German female & 14 & 26.929 & 7 & 43 & 10.680 \\
\hline $\begin{array}{c}\text { Male vs. female diff. } \\
\text { Canada vs. Germany } \\
\text { diff. }\end{array}$ & - & $\begin{array}{l}1.055 \\
(0.873)\end{array}$ & - & - & - \\
\hline $\begin{array}{c}\text { C1.688 } \\
(0.001)\end{array}$ & - & - & - \\
\hline $\begin{array}{c}\text { Male vs. female diff. }- \\
\text { Canada }\end{array}$ & - & $\begin{array}{l}-7.125 \\
(\mathbf{0 . 5 2 2})\end{array}$ & - & - & - \\
\hline $\begin{array}{c}\text { Male vs. female diff. - } \\
\text { Germany }\end{array}$ & - & $\begin{array}{l}12.127 \\
(0.012)\end{array}$ & - & - & - \\
\hline $\begin{array}{c}\text { Canada vs. Germany - } \\
\text { males }\end{array}$ & - & $\begin{array}{l}12.819 \\
(0.122)\end{array}$ & - & - & - \\
\hline $\begin{array}{c}\text { Canada vs. Germany - } \\
\text { females }\end{array}$ & - & $\begin{array}{l}32.071 \\
(0.001)\end{array}$ & - & - & - \\
\hline
\end{tabular}




\section{Review of findings up to}

now

口 Overconfidence:

- Individuals are overconfident.

- No difference between genders.

- Canadian students more OC.

Trading activity:

- No difference between genders.

- Canadian students trade more.

$\square$ Canadian vs. German differences in line with theory. 


\section{Trading activity: German vs}

\section{Canadian females:}

$\square$ Canadian females trade as much as males (actually more).

$\square$ German females trade significantly less than males.

$\square$ Why?

$\square$ An inhibition effect? 
Table 5: Regressions of trading activity on calibration-based overconfidence for various subgroups

This table displays the coefficients and p-values when trading activity (number of trades during the 12 single-period markets) is regressed on calibration-based overconfidence (OC). OC is the calibration-based metric for overconfidence with high values indicating high levels of overconfidence. Results reported use heteroskedasticity-consistent standard errors.

Trading activity $=\mathbf{C ( 1 )}+\mathbf{C ( 2 )} \mathrm{OC}$

\begin{tabular}{|l|l|l|l|l|}
\hline Group & OBS & $\mathbf{C ( 1 )}$ & $\mathbf{C ( 2 )}$ & $\mathbf{R}^{\mathbf{2}}$ \\
\hline All & 64 & $\begin{array}{c}4.119 \\
(0.755)\end{array}$ & $\begin{array}{l}51.808 \\
(0.007)\end{array}$ & $7.7 \%$ \\
\hline Male & 34 & $\begin{array}{c}3.934 \\
(0.794)\end{array}$ & $\begin{array}{l}53.714 \\
(0.019)\end{array}$ & $12.3 \%$ \\
\hline Female & 30 & $\begin{array}{c}2.979 \\
(0.912)\end{array}$ & $\begin{array}{l}51.426 \\
(0.161)\end{array}$ & $4.5 \%$ \\
\hline Canadian & 32 & $\begin{array}{l}-34.552 \\
(0.667)\end{array}$ & $\begin{array}{l}106.852 \\
(0.275)\end{array}$ & $3.7 \%$ \\
\hline Canadian male & 16 & $\begin{array}{l}-83.000 \\
(0.520)\end{array}$ & $\begin{array}{l}159.262 \\
(0.317)\end{array}$ & $7.5 \%$ \\
\hline Canadian female & 16 & $\begin{array}{l}-4.810 \\
(0.967)\end{array}$ & $\begin{array}{l}76.190 \\
(0.586)\end{array}$ & $2.1 \%$ \\
\hline German & 32 & $\begin{array}{l}24.500 \\
(0.027)\end{array}$ & $\begin{array}{l}12.841 \\
(0.378)\end{array}$ & $2.5 \%$ \\
\hline German male & 18 & $\begin{array}{l}14.387 \\
(0.252)\end{array}$ & $\begin{array}{l}35.522 \\
(0.040)\end{array}$ & $21.7 \%$ \\
\hline German female & 14 & $\begin{array}{l}34.771 \\
(0.063)\end{array}$ & $\begin{array}{l}-10.408 \\
(0.618)\end{array}$ & $2.2 \%$ \\
\hline
\end{tabular}


Table 6: Regressions of average wealth on calibration-based overconfidence and prediction error

This table displays the coefficients and p-values when trading success (average wealth) is regressed on calibration-based overconfidence (OC) and prediction error. High values of $\mathrm{OC}$ indicate high levels of overconfidence. The prediction error is given by the sum of absolute differences between the individual's dividend predictions and the dividend realizations over the 12 markets. The regression is run either including all markets or excluding the first, the first two, the first three or the first four markets. Results reported use heteroskedasticityconsistent standard errors.

$$
\text { Average Wealth }=\mathrm{C}(1)+\mathrm{C}(2) \mathrm{OC}+\mathrm{C}(3) \text { Prediction Error }
$$

\begin{tabular}{|l|l|l|l|l|l|}
\hline $\begin{array}{l}\text { Excluded } \\
\text { markets }\end{array}$ & OBS & C(1) & C(2) & C(3) & $\mathbf{R}^{2}$ \\
\hline None & 64 & $\begin{array}{l}430.453 \\
(0.000)\end{array}$ & \begin{tabular}{llll|}
-28.752 \\
$(0.1832)$
\end{tabular} & $\begin{array}{l}-0.021 \\
(0.1977)\end{array}$ & $1.9 \%$ \\
\hline 1 & 64 & $\begin{array}{l}438.720 \\
(0.000)\end{array}$ & $\begin{array}{l}-38.241 \\
(0.078)\end{array}$ & $\begin{array}{l}-0.031 \\
(0.072)\end{array}$ & $3.2 \%$ \\
\hline $1 \& 2$ & 64 & $\begin{array}{l}446.502 \\
(0.000)\end{array}$ & $\begin{array}{l}-56.493 \\
(0.003)\end{array}$ & $\begin{array}{l}-0.036 \\
(0.013)\end{array}$ & $7.1 \%$ \\
\hline $1,2 \& 3$ & 64 & $\begin{array}{l}435.503 \\
(0.000)\end{array}$ & $\begin{array}{l}-49.027 \\
(0.017)\end{array}$ & $\begin{array}{l}-0.036 \\
(0.036)\end{array}$ & $5.4 \%$ \\
\hline $1,2,3 \& 4$ & 64 & $\begin{array}{l}460.118 \\
(0.000)\end{array}$ & $\begin{array}{l}-74.155 \\
(0.003)\end{array}$ & $\begin{array}{l}-0.045 \\
(0.039)\end{array}$ & $7.6 \%$ \\
\hline
\end{tabular}




\section{Trading and performance as a function of overconfidence}

$\square$ Overconfidence leads to more trade.

- Especially males.

- And German males.

$\checkmark$ Performance deteriorates with overconfidence.

- Especially after first couple of markets are dropped. 


\section{Table 7: Correlation matrix of overconfidence measures}

$\mathrm{OC}$ is the calibration-based metric for overconfidence, with high values indicating high levels of overconfidence. A well-calibrated individual would have $90 \%$ of the correct answers contained inside her intervals, leading to $\mathrm{OC}=0.1$. BTA is the better-than-average metric for overconfidence, where high values indicate high levels of overconfidence. BTA=15.5 indicates neither overconfidence nor underconfidence. Finally, IOC is the illusion of control metric for overconfidence, where high levels indicate high overconfidence. A value above zero indicates overconfidence. The maximum (minimum) value of IOC is by construction $4(-4)$. In the lower panel of the table below cross-correlations and (in brackets) p-values are reported. The upper panel displays the mean, standard deviation (SD) and the maximum and minimum values of these overconfidence measures.

\begin{tabular}{|c|c|c|c|}
\hline & OC & BTA & IOC \\
\hline Mean & 0.781 & 15.744 & 0.469 \\
\hline SD & 0.1402 & 5.722 & 1.642 \\
\hline Maximum & 1.000 & 31.000 & 4.000 \\
\hline Minimum & 0.450 & 0.000 & -4.000 \\
\hline \multicolumn{4}{|c|}{ Correlation Matrix } \\
\hline & $\mathbf{O C}$ & BTA & IOC \\
\hline $\mathbf{O C}$ & 1 & $\begin{array}{l}.119 \\
(0.348) \\
\end{array}$ & $\begin{array}{l}.091 \\
(0.477) \\
\end{array}$ \\
\hline BTA & & 1 & $\begin{array}{l}.212 \\
(0.092)\end{array}$ \\
\hline IOC & & & 1 \\
\hline
\end{tabular}


Table 8: Regressions of trading activity on various overconfidence metrics

This table displays the coefficients and their p-values from regressions when trading activity is regressed on various measures of overconfidence. OC is the calibration-based metric for overconfidence with high values indicating high levels of overconfidence. BTA is the better-than-average metric for overconfidence where high values indicate high levels of overconfidence. IOC is the illusion of control metric for overconfidence where high levels indicate high overconfidence. Results reported use heteroskedasticity-consistent standard errors. Adjusted $\mathbf{R}^{2}$ is in parentheses below $\mathbf{R}^{2}$.

Trading activity $=\mathbf{C ( 1 )}+\mathbf{C ( 2 )}$ OC $+\mathrm{C}(3) \mathrm{BTA}+\mathrm{C}(4) \mathrm{IOC}$

\begin{tabular}{|l|l|l|l|l|l|}
\hline OBS & C(1) & $\mathbf{C ( 2 )}$ & $\mathbf{C ( 3 )}$ & $\mathbf{C ( 4 )}$ & $\mathbf{R}^{2}$ \\
\hline 64 & -8.854 & 49.673 & 1.008 & -2.611 & $13.8 \%$ \\
& $(0.509)$ & $(0.008)$ & $(0.027)$ & $(0.152)$ & $(9.5 \%)$ \\
\hline 64 & -6.085 & 47.650 & 0.854 & & $11.2 \%$ \\
& $(0.671)$ & $(0.014)$ & $(0.062)$ & & $(8.3 \%)$ \\
\hline
\end{tabular}


Table 9: Regressions of trading activity on overconfidence metrics and demographic variables

This table displays the coefficients and p-values from regressions when trading activity is regressed on calibration-based overconfidence (OC), better than average-based overconfidence (BTA), age (AGE), financial education (EDUC), mutual fund exposure (MUT), trading experience (EXP), calibration session knowledge (KNOW), country (COUNTRY) and gender (GENDER). Note that high values of OC and high values of BTA indicate high levels of overconfidence. Further note that COUNTRY=1 for Canada and GENDER=1 for male. Results reported use heteroskedasticity-consistent standard errors. Adjusted $\mathbf{R}^{2}$ is in parentheses below $\mathbf{R}^{2}$.

\begin{tabular}{|c|c|c|c|c|}
\hline $\begin{array}{l}\text { IND. } \\
\text { VAR. }\end{array}$ & (1) & (2) & (3) & (4) \\
\hline Constant & \begin{tabular}{|l|}
43.908 \\
$(0.026)$
\end{tabular} & $\begin{array}{l}51.748 \\
(0.004)\end{array}$ & $\begin{array}{l}31.709 \\
(0.058)\end{array}$ & \begin{tabular}{|l|}
38.815 \\
$(0.040)$
\end{tabular} \\
\hline $\mathbf{O C}$ & $\begin{array}{l}40.499 \\
(0.023) \\
\end{array}$ & $\begin{array}{l}45.489 \\
(0.003) \\
\end{array}$ & $\begin{array}{l}29.964 \\
(0.041)\end{array}$ & \begin{tabular}{|l|}
28.854 \\
$(0.063)$ \\
\end{tabular} \\
\hline BTA & \begin{tabular}{|c|}
0.700 \\
$(0.164)$ \\
\end{tabular} & & & \\
\hline AGE & \begin{tabular}{|c|}
-1.818 \\
$(0.016)$ \\
\end{tabular} & \begin{tabular}{|l|}
-1.923 \\
$(0.008)$ \\
\end{tabular} & \begin{tabular}{|c|}
-0.872 \\
$(0.205)$ \\
\end{tabular} & $\begin{array}{l}-0.997 \\
(.1644)\end{array}$ \\
\hline EDUC & \begin{tabular}{|l|}
-0.794 \\
$(0.003)$ \\
\end{tabular} & $\begin{array}{l}-0.717 \\
(0.004)\end{array}$ & \begin{tabular}{|l|}
-0.454 \\
$(0.048)$ \\
\end{tabular} & $\begin{array}{l}-0.525 \\
(0.040) \\
\end{array}$ \\
\hline MUT & $\begin{array}{l}-1.241 \\
(0.824)\end{array}$ & & & \\
\hline EXP & \begin{tabular}{|l|}
16.454 \\
$(0.023)$ \\
\end{tabular} & \begin{tabular}{|l|l}
17.991 \\
$(0.010)$ \\
\end{tabular} & \begin{tabular}{|l|}
17.060 \\
$(0.014)$ \\
\end{tabular} & \begin{tabular}{|l|}
20.818 \\
$(0.003)$ \\
\end{tabular} \\
\hline KNOW & \begin{tabular}{|c|}
0.003 \\
$(0.971)$ \\
\end{tabular} & & & \\
\hline COUNTRY & & & \begin{tabular}{|l|}
13.756 \\
$(0.028)$ \\
\end{tabular} & \begin{tabular}{|c|}
12.952 \\
$(0.046)$ \\
\end{tabular} \\
\hline GENDER & & & & \begin{tabular}{|l|}
-7.004 \\
$(0.244)$ \\
\end{tabular} \\
\hline $\mathbf{R}^{2}$ & $\begin{array}{l}27.5 \% \\
(18.5 \%) \\
\end{array}$ & $\begin{array}{l}25.3 \% \\
(20.2 \%) \\
\end{array}$ & $\begin{array}{l}28.6 \% \\
(22.5 \%)\end{array}$ & $\begin{array}{l}30.0 \% \\
(22.6 \%)\end{array}$ \\
\hline
\end{tabular}




\section{BTA, IOC and other factors}

\section{explaining trading}

$\square$ Calibration-based OC predominates.

- BTA seems to matter as well.

$\square$ Once demographics are brought in, even BTA does not matter.

- Less educated people trade more.

- Experienced traders trade more.

- Country also matters.

$\square$ Gender has no impact. 
This table displays the coefficients and p-values (in parentheses) when generalized least squares regressions are done with heteroscedastic errors across trading sessions and autocorrelated errors across markets. The first two rows show regression results for both German and Canadian data where the market trading volume in session $i$ at market $t$ (denoted as VOLUME(i,t)) is regressed on the average calibration-based overconfidence level of the eight traders in each session (denoted as MEANOC(i)) and (potentially) a country dummy (denoted as COUNTRY(i) where COUNTRY(i) $=1$ for Canada and zero otherwise). The third row reports the regression results for German data where market trading volume (VOLUME( $i, t)$ ) is regressed on an overconfidence level indicator variable (denoted as $\mathrm{H}(\mathrm{i})$, where $(\mathrm{H}(\mathrm{i}))=1$ for a low level of overconfidence and zero otherwise. The fourth row displays the regression results for Canadian data where market trading volume (VOLUME(i,t)) is regressed on a gender dummy variable (denoted as $G(\mathrm{i})$, where $\mathrm{G}(\mathrm{i})=1$ for an all-male session and zero otherwise). Analogous to ordinary least squares, for generalized least squares the Buse (1973) $R^{2}$ can be interpreted as a measure of the proportion of the generalized sums of squares of the dependent variable which is attributable to the influence of the explanatory variables.

For trading session $i$ in market $t$ :

VOLUME(i,t)= C(1) + C(2)COUNTRY(i)+C(3) MEANOC(i) + C(4) H(i) $+\mathbf{C}(5) \mathrm{G}(\mathbf{i})+\mathbf{e}(\mathbf{i}, \mathbf{t})$

where $e(i, t)=\operatorname{rho}(i) e(i, t-1)+v(i, t)$ with $E[v(i, t)]=0$; and $E[v(i, t) v(j, s)]=v a r i a n c e(i)$ for $i=j, t=s ;$ otherwise $E[v(i, t) v(j, s)]=0$.

\begin{tabular}{|c|c|r|c|r|r|r|c|}
\hline Group & Obs & C(1) & C(2) & C(3) & C(4) & C(5) & $\begin{array}{c}\text { Buse } \\
\mathbf{R}^{2}\end{array}$ \\
\hline $\begin{array}{c}\text { All sessions } \\
\text { (no country } \\
\text { indicator) }\end{array}$ & 96 & $\begin{array}{r}5.297 \\
(0.201)\end{array}$ & & $\begin{array}{r}8.486 \\
(0.145)\end{array}$ & & & $2.24 \%$ \\
\hline $\begin{array}{c}\text { All sessions } \\
\text { (with country } \\
\text { indicator) }\end{array}$ & 96 & $\begin{array}{r}3.762 \\
(0.266)\end{array}$ & $\begin{array}{r}7.559 \\
(0.003)\end{array}$ & $\begin{array}{r}9.882 \\
(0.020)\end{array}$ & & & $14.96 \%$ \\
\hline $\begin{array}{c}\text { German } \\
\text { sessions }\end{array}$ & 48 & $\begin{array}{r}12.445 \\
(0.000)\end{array}$ & & & $\begin{array}{r}-3.107 \\
(0.022)\end{array}$ & & $10.83 \%$ \\
\hline $\begin{array}{c}\text { Canadian } \\
\text { sessions }\end{array}$ & 48 & $\begin{array}{r}15.249 \\
(0.001)\end{array}$ & & & & $\begin{array}{r}5.329 \\
(0.346)\end{array}$ & $1.94 \%$ \\
\hline
\end{tabular}




\section{Conclusions}

$\square$ Hypothesis 1 is supported:

- OC traders trade more.

- Miscalibration is predominant.

- OC matters both at individual and market level.

- Other effects matter. 


\section{Conclusions cont.}

$\square$ Hypothesis 2: Controlling for overconfidence, women trade less than men.

- Men and women are equally OC.

- Men and women trade the same amount.

- In a regression of trading on OC gender does not have an incremental impact. 


\title{
Thanks. Questions?
}

\author{
Richard Deaves \\ deavesr@mcmaster.ca
}

